\title{
Un nouveau départ: deux mètres à la fois
}

\author{
Douglas McDougall ${ }^{1}$
}

Accepted: 27 September 2021 /Published online: 27 October 2021

(C) Ontario Institute for Studies in Education (OISE) 2021

Il y a un certain nombre de moments dans l'année que l'on considère comme de «nouveaux» départs. Le moment qui représente pour moi le nouveau départ le plus palpitant est celui de septembre (dans certains territoires, provinces ou régions, l'année scolaire débute en août ou en janvier, mais tous ces départs restent passionnants pour moi). À cette période de l'année, il existe un fort potentiel de nouvelles expériences. La nouveauté se manifeste partout: les étudiants dans nos cours, les cours que nous donnons, les rôles que nous jouons en éducation, nos questions de recherche et nos projets de rédaction.

Le numéro 21.3 de la RCESMT célèbre aussi le nouveau et l'ancien qui se renouvellent. Nous avons relancé dans la revue la rubrique «L'actualité en bref». Cette section, publiée durant les premières années suivant la création de la revue avait pour but de partager les nouvelles et faire connaître les événements au sein de la communauté des SMT. À la suite de réflexions formulées par les directeurs de rédaction précédents (Blades, 2020) et des discussions faites avec les rédacteurs en chefs actuels, nous avons réintégré la rubrique. Le $\mathrm{p}^{\mathrm{r}}$ Alexandre Cavalcante de l'Institut d'études pédagogiques de l'Ontario attaché à l'Université de Toronto est l'éditorialiste en chef de «L'actualité en bref». Sa chronique inaugurale est publiée dans ce numéro. Il y a aussi une nouveauté appelée «Canadian Mathematics Education Matters (Les enjeux de l'enseignement canadien des mathématiques)» publiée par le rédacteur en chef pour les mathématiques, Egan Chernoff. Dans son premier article, «The Metre as a Metric: Canada's COVID-19 Conversion Kerfuffle (La métrique du mètre: le cafouillage canadien de la conversion liée à la COVID19)», il partage de façon humoristique ses observations sur la signalétique qui attribue une équivalence entre six pieds et deux mètres pour indiquer les exigences gouvernementales et organisationnelles en matière de distanciation sociale (Cernoff, 2021). Il démontre par écrit et sous forme de représentation graphique combien au Canada on représente le système métrique de façon maladroite durant la pandémie mondiale de COVID-19.

L'un des thèmes abordés dans ce numéro porte sur la création et l'usage des manuels de science. Kim et al. (2021) conduisent des entrevues avec des auteurs canadiens de manuels de science dans le but de mieux comprendre leurs attentes concernant les manuels et certains des défis qu'ils

Douglas McDougall

doug.mcdougall@utoronto.ca

1 Ontario Institute for Studies in Education, University of Toronto, 252 Bloor Street West, Toronto, ON M5S 1V6, Canada 
ont rencontrés. Les auteurs de manuels décrivent combien il a été difficile pour eux d'aborder les enjeux sociaux dans leurs ouvrages en raison de la résistance des enseignants en sciences. L'une des principales attentes était que les enseignants développent et utilisent d'autres sources, y compris des manuels, qu'ils s'engagent dans des discussions sur les enjeux socio-environnementaux plutôt que de se fier uniquement aux manuels de science. Kim et al. (2021) avancent qu'il est très difficile de transformer le discours des connaissances scientifiques dans les manuels, mais qu'ils demeurent avec l'espoir que la volonté humaine finira par changer les choses.

Ndumanya et al. (2021) examinent les manuels utilisés en Afrique du Sud. Leur étude, qui vise à mieux cerner l'étendue des pratiques fondées sur la recherche dans les manuels, se concentre sur les manuels scientifiques de trois écoles. Les chercheurs constatent que les activités et les pratiques scientifiques présentées dans les manuels s'avèrent surtout dirigées par l'enseignant et qu'elles ne donnent lieu qu'à très peu d'occasions pour l'élève de participer activement aux pratiques scientifiques. Ils établissent aussi la nécessité de modifier les manuels afin de représenter ces pratiques. Ces deux articles montrent combien il est difficile de changer les manuels de science. Comme les manuels représentent souvent le "programme éducatif» des enseignants, cette étude joue un rôle très important alors que de nouveaux manuels sont rédigés et que les anciens sont mis à jour selon notre compréhension de la dynamique impliquée dans l'apprentissage par les élèves du contenu scientifique et des pratiques qui y sont associées.

Dans le but d'apprendre sur les expériences des étudiants en communication orale concernant la science, Langlois et al. (2021) ont conduit une étude auprès d'étudiants de niveau collégial. Ceux-ci ont rempli un questionnaire mesurant leurs perceptions et attitudes à l'égard de la communication orale en science et 26 répondants ont de plus été interviewés. Les chercheurs ont constaté qu'audelà des normes, du contenu et de la capacité à présenter sur un sujet scientifique, la dépendance contextuelle constituait un élément important à être ajouté au modèle de van Aalderen-Smeets et coll. (2012). Ils ont établi d'autres aspects qui s'avèrent des sous-éléments attitudinaux à l'égard de la communication orale concernant la science comme l'anxiété, le plaisir et la perception quant à la pertinence.

Dans une classe composée d'élèves de cinquième et de sixième année, Jin et Kim (2021) examinent les conceptions épistémiques des élèves du primaire à l'égard de l'argumentation scientifique. Les chercheurs ont observé ce qui se déroulait dans la classe, ont recueilli des échantillons de travaux faits par les élèves et ont interviewé 19 élèves et leur enseignant. Il a été démontré que les élèves étaient conscients de leurs conceptions épistémiques et que celles-ci étaient peaufinées dans les interactions sociales et une exposition à de nouveaux contextes. Jin et Kim (2021) ont aussi constaté que la performance des élèves n'était pas uniforme et que certains d'entre eux ne mettaient pas en pratique leurs conceptions épistémiques, même lorsqu'ils en étaient pleinement conscients. Les enseignants qui engagent leurs élèves dans la pratique argumentative et des conversations explicites concernant l'argumentation peuvent contribuer à améliorer la conception épistémique des élèves et leurs compétences en argumentation.

Martin et al. (2021) ont fait un sondage auprès de 626 enseignants pour mieux comprendre les pratiques d'enseignement des probabilités aux élèves du primaire et du secondaire. Les chercheurs se sont penchés sur le niveau d'éducation, le degré d'aptitude en mathématiques, le niveau de confiance à l'égard de leur enseignement, le recours planifié aux approches probabilistes et l'usage de matériel de manipulation. Les résultats de l'étude montrent que ces enseignants ont rapporté un niveau de confiance didactique moindre en ce qui concerne l'enseignement des probabilités au primaire et au secondaire en comparaison avec les autres sujets mathématiques, sauf pour l'enseignement des statistiques au niveau secondaire. Au fur et à mesure que nous améliorons nos connaissances sur la confiance des enseignants, les auteurs avancent que les observations faites en classe sur l'enseignement 
des probabilités serviront à enrichir notre compréhension et aideront à mieux cerner les pratiques enseignantes dans les écoles primaires et secondaires.

Gómez-Blancarte et Miranda (2021) se penchent sur l'élaboration d'un programme professionnel de mathématiques concernant les statistiques auquel ont participé cinq enseignants du secondaire actuellement en service. Ils utilisent deux concepts, la participation et la réification en tant que dimensions du processus de conception pédagogique. Les auteurs expliquent comment ces deux dimensions interagissent afin de permettre aux enseignants de poursuivre leur apprentissage au sein du programme de développement professionnel. Les concepteurs du programme encouragent la communication entre les chercheurs du domaine de l'enseignement des statistiques et les enseignants des mathématiques afin d'entamer une discussion sur la création de plans de cours et pour utiliser les salles de classe des enseignants dans le but d'apprendre quelles sont les pratiques requises afin d'intégrer ces plans de cours. Les auteurs proposent une étude plus poussée sur la participation et la réification en contexte de pratique enseignante comme une avenue à envisager.

Proulx et Mégrourèche (2021) décrivent leurs expérimentations d'enseignement dans le cadre de leur étude sur les stratégies mathématiques employées par les élèves dans les salles de classe au primaire et au secondaire. Les enseignants impliqués dans l'étude ont souligné maintes fois l'impact positif de la dynamique de classe, plus particulièrement l'instauration d'un climat qui encourage l'engagement des élèves. Les auteurs ont recours au concept de bienveillance pour conceptualiser l'impact des expérimentations d'enseignement sur les élèves, l'atmosphère de la classe et sur la nature même des mathématiques. Ils fournissent au lecteur des aperçus concernant les productions mathématiques des élèves et sur l'enseignement en classe.

Jeannotte et al. (2021) se penchent sur le savoir et les pratiques d'enseignement des enseignants du primaire pour favoriser le développement du raisonnement mathématique au Québec. Par l'analyse du discours, les auteures soulignent des mots-clés, des habitudes communes et des récits concernant la pratique enseignante. Elles indiquent qu'il y a deux façons de concevoir le raisonnement mathématique: en tant que compétence à évaluer ou en tant que processus de compréhension. Ces deux façons mènent à des fondements et à des règles métadiscursives qui ne sont pas les mêmes, ce qui donne lieu à des routines différentes et amène des conflits pour les personnes de cette communauté. Les auteures proposent que le programme des mathématiques soit changé afin d'aider les enseignants du primaire à élargir leur façon de concevoir et de favoriser le raisonnement mathématique dans la salle de classe.

Setyowati et al. (2021) présentent une critique de l'ouvrage intitulé «STEM Education across the Learning Continuum: Early Childhood to Senior Secondary (L'enseignement des STIM dans tout le continuum de l'apprentissage: De la petite enfance au niveau secondaire de deuxième cycle)» (MacDonald et al., 2020). Ils indiquent qu'on y retrouve des perspectives et des contextes issus de pays comme l'Australie, la Suède le Royaume-Uni, le Qatar et les États-Unis. Par une série d'études empiriques (neuf chapitres) et de documents conceptuels (six chapitres), l'ouvrage fournit aux chercheurs, aux enseignants et aux administrateurs des moyens pour intégrer les STIM à l'éducation offerte à partir de la petite enfance jusqu' au niveau secondaire.

La rédaction et le personnel de la RCESMT souhaitent que la nouvelle année scolaire apporte de nouvelles perspectives, de l'énergie, des questionnements et de l'émerveillement à vos vies professionnelles et personnelles. Nous savons qu'il reste beaucoup d'incertitude alors que nous nous adaptons face aux changements causés par la pandémie de COVID-19. È mesure que nous progressons en tant que communauté, j'ose espérer que la possibilité de vous épanouir au niveau professionnel et vos objectifs universitaires sauront vous motiver à partager vos nouvelles connaissances avec nous et avec autrui par l'entremise de revues professionnelles et spécialisées. 


\section{References}

Blades, D. (2020). Newsround: A repetition. Canadian Journal for Science, Mathematics and Technology, 20(4), $780-783$. Chernoff, E. (2021). The metre as a metric: Canada's COVID-19 conversion kerfuffle. Canadian Journal of Science, nMathematics and Technology Education, 21(3). https://doi.org/10.1007/s42330-021-00176-0

Gómez-Blancarte, A. L. \& Miranda, I. (2021). Participation and reification: two basic design principles for mathematics professional development programs. Canadian Journal of Science, Mathematics and Technology Education, 21(3). https://doi.org/10.1007/s42330-021-00175-1

Jeannotte, D., Sampson, S., \& Dufour, S. (2021). Caractérisation du discours mathématique pour l'enseignement associé au raisonnement mathématique d'enseignantes du primaire au Québec: une exploration. Canadian Journal of Science, Mathematics and Technology Education, 21(3). https://doi.org/10.1007/s42330-021-00165-3

Jin, Q. \& Kim, M. (2021). Elementary students' epistemic understandings in their classroom scientific argumentation. Canadian Journal of Science, Mathematics and Technology Education, 21(3). https://doi.org/10.1007/ s42330-021-00166-2

Kim, M., Wagner, D., \& Jin, Q. (2021). Tensions and hopes for embedding peace and sustainability in science education: stories from science textbook authors. Canadian Journal of Science, Mathematics and Technology Education, 21(3). https://doi.org/10.1007/s42330-021-00157-3

Langlois, S., Cormier, C, \& Marec, C-É. (2021). Respecter les normes et posséder un sens du spectacle en communication orale en sciences : les deux aspects du sentiment d'efficacité personnelle d'étudiants postsecondaires en sciences Canadian Journal of Science, Mathematics and Technology Education, 21(3). https://doi.org/10.1007/s42330-02100170-6

Martin, V., Thibault, M., \& Roy, N. (2021). Pratiques déclarées d'enseignement des probabilités : enquête auprès de personnes enseignantes du primaire et secondaire au Québec. Canadian Journal of Science, Mathematics and Technology Education, 21(3). https://doi.org/10.1007/s42330-021-00177-z

MacDonald, A., Danaia, L., \& Murphy, S. (Eds.) (2020). STEM Education across the Learning Continuum: Early Childhood to Senior Secondary. Springer.

Ndumanya, E., Ramnarain, U., \& Wu, H.-K. (2021). An analysis of selected grade 12 physical sciences textbooks for the inclusion of the NGSS science practices. Canadian Journal of Science, Mathematics and Technology Education, 21(3). https://doi.org/10.1007/s42330-021-00169-z

Proulx, J. \& Mégrourèche, C. (2021). Retombées collatérales d'un Teaching Experiment : vers une bienveillance didactique. Canadian Journal of Science, Mathematics and Technology Education, 21(3). https://doi.org/10.1007/ s42330-021-00171-5

Setyowati, Y., Firda, R., \& Kasmita, W. (2021). STEM education: exploring practices across education levels. Canadian Journal of Science, Mathematics and Technology Education, 21(3). https://doi.org/10.1007/s42330-021-00172-4

van Aalderen-Smeets, S. I., Walma van der Molen, J. H., \& Asma, L. J. F. (2012). Primary teachers' attitudes toward science: A new theoretical framework. Science Education, 96(1), 158-182. https://doi.org/10.1002/sce.20467

Publisher's Note Springer Nature remains neutral with regard to jurisdictional claims in published maps and institutional affiliations. 